\title{
Reliability and Validity of the Korean Version of the Childhood Trauma Questionnaire-Short Form for Psychiatric Outpatients
}

\author{
Daeho Kim ${ }^{1,2} \bowtie$, Seon-Cheol Park ${ }^{1,2}$, Hyunjoo Yang ${ }^{1}$ and Dong Hoon Oh${ }^{1}$ \\ ${ }^{1}$ Department of Neuropsychiatry, Hanyang University Medical School, Seoul, Korea \\ ${ }^{2}$ Traumatic Stress Clinic, Department of Psychiatry, Hanyang University Guri Hospital, Guri, Korea
}

Objective The Childhood Trauma Questionnaire (CTQ) is perhaps the most widely used and well-studied retrospective measure of childhood abuse or neglect. This study tested the initial reliability and validity of a Korean translation of the Childhood Trauma Questionnaire (CTQ-K) among non-psychotic psychiatric outpatients.

Methods The CTQ-K was administered to a total of 163 non-psychotic psychiatric outpatients at a university-affiliated training hospital. Internal consistency, four-week test-retest reliability, and validity were calculated. A portion of the participants (n=65) also completed the Trauma Assessment Questionnaire (TAQ), the Impact of Events Scale-Revised, and the Dissociative Experiences Scale-Taxon.

Results Four-week test-retest reliability was high ( $\mathrm{r}=0.87$ ) and internal consistency was good (Cronbach's $\alpha=0.88$ ). Each type of childhood trauma was significantly correlated with the corresponding subscale of the TAQ, thus confirming its concurrent validity. In addition, the CTQ-K total score was positively related to post-traumatic symptoms and pathological dissociation, demonstrating the convergent validity of the scale. The CTQ-K was also negatively correlated with the competence and safety subscale of the TAQ, confirming discriminant validity. Additionally, we confirmed the factorial validity by identifying a five-factor structure that explained $64 \%$ of the total variance.

Conclusion Our study indicates that the CTQ-K is a measure of psychometric soundness that can be used to assess childhood abuse or neglect in Korean patients. It also supports the cross-cultural equivalence of the scale.

Psychiatry Investig 2011;8:305-311

Key Words Childhood trauma questionnaire, Reliability and validity, Trauma, Child abuse, Culture.

\section{INTRODUCTION}

It is not likely that psychiatric patients voluntarily disclose their past history of child abuse, ${ }^{1}$ nor do clinicians tend to ask specifically about childhood trauma in their routine assessments. ${ }^{2,3}$ This is an unsatisfactory situation given that the adult survivors of childhood abuse are over-represented among psychiatric patients. ${ }^{4}$ Studies of psychiatric outpatients have found that $48-68 \%$ of those sampled reported childhood physical or sexual abuse. ${ }^{5,6}$ Research has also shown that childhood abuse plays a pivotal role in development and influences the manifestation and course of many

Received: February 11, 2011 Revised: August 20, 2011

Accepted: August 23, 2011 Available online: November 25, 2011

$\triangle$ Correspondence: Daeho Kim, MD, PhD

Department of Psychiatry, Hanyang University Guri Hospital, 249-1 Gyomundong, Guri 471-701, Korea

Tel: +82-31-560-2274, Fax: +82-31-554-2599

E-mail: dkim9289@hanyang.ac.kr

(c) This is an Open Access article distributed under the terms of the Creative Commons Attribution Non-Commercial License (http://creativecommons.org/licenses/by$\mathrm{nc} / 3.0$ ) which permits unrestricted non-commercial use, distribution, and reproduction in any medium, provided the original work is properly cited. psychiatric illnesses including post-traumatic stress disorder, dissociative disorder, depression, anxiety disorder, substance abuse, personality disorder, and psychosis. ${ }^{7}$

Therefore, identifying childhood trauma in initial assessments is crucial for further planning of the management and treatment planning of psychiatric patients. One way to achieve this goal is to include specific behavioral questions about childhood adversities during the routine intake interview; another practical way is to use questionnaires and to discuss their responses with the patients. ${ }^{8}$

Of the retrospective self-report measures for childhood trauma, the Childhood Trauma Questionnaire (CTQ) developed by Bernstein et al. ${ }^{9}$ has undergone the most extensive psychometric testing in both clinical and general populations. ${ }^{10}$ This tool has also been used in numerous studies, some of which have examined the validity and reliability of different language versions. ${ }^{11-13}$ In addition to a rich literature of support for its psychometric properties and the norms it has established, the CTQ has other merits in that it is short (5-10 minutes to complete), measures five types of childhood maltreat- 
ment, and is designed for both adults and adolescents. ${ }^{14}$

The CTQ evaluates five domains of childhood abuse and neglect: sexual abuse (SA), physical abuse (PA), emotional abuse (EA), physical neglect (PN), and emotional neglect (EN). The original CTQ contained 70 items. However, it was later reduced to a 28-item short form (CTQ-SF), which has also been psychometrically validated..$^{15}$ Overall, the literature on the CTQ supports its construct validity, including its internal consistency and factorial stability, although some concern exists about its validity for PN. However, a few studies have reported its concurrent validity with other similar selfreport measures.

This study examined the reliability and validity of a Korean translation of the CTQ-SF and its subscales for non-psychotic psychiatric patients. Specifically, we measured four-week test-retest reliability and concurrent validity with a similar self-report measure because these psychometric properties have not been addressed in previous studies.

\section{METHODS}

\section{Participants}

All participants were recruited from the outpatient Department of Psychiatry at Hanyang University Guri Hospital, Gyeonggi, South Korea. Patients were considered eligible to enter the study if 1 ) they were given a DSM-IV diagnosis by the psychiatric specialist, 2) they were between the ages of 18 and 64, and 3) were able to read and write. Patients were excluded if they had 1) any psychotic illness, 2) an IQ less than 80 , or 3) a cognitive or neurological disorder.

The CTQ-K data with other questionnaires were gathered from a consecutive sample of psychiatric outpatients recruited by two psychiatrists (DK and SCP) over a one-month period. Initial screening identified 78 patients who satisfied these criteria; however, 13 patients (20\%) refused to participate, and the final sample consisted of 65 participants. Those who refused to enter the study were not significantly different from the participants with regard to age, sex, psychiatric diagnosis, or education. In addition, the CTQ-K was verified in a non-random sample of 40 patients (62\%) after four weeks (mean=29.0 days, $\mathrm{SD}=8.0$ ) to assess the short-term test-retest reliability.

To achieve a reasonable sample size for factor analysis, the results of the CTQ-K from an additional 98 outpatients were included. This second set of data was from a convenient sample and did not include data from other instruments.

The total sample of 163 participating patients were predominantly women $(61 \%)$ and had a mood or anxiety disorder (66\%), high school diploma (62\%) and monthly income less than 4 million won (74\%). Approximately half of the sample was married (53\%), employed (46\%), and not religiously affiliated (46\%). The sample had a mean age of 38.3 years $(\mathrm{SD}=11.8)$ with a range of 18 to 64 years (Table 1$)$.

\section{Procedure}

During a formal consultation, the eligible participants were approached by a psychiatrist (either DK or SCP), who fully described the nature and intent of the study and obtained written informed consent. Those who consented were given either the CTQ-K or a questionnaire including the CTQ-K, the Trauma Antecedents Questionnaire (TAQ), ${ }^{16}$ the Impact of Events Scale-Revised (IES-R), ${ }^{17}$ and the Dissociative Experiences Scale-Taxon (DES-T).$^{18}$ In addition, clinical and sociodemographic information was obtained from patients and their medical records. This study was approved by the institutional review board of Hanyang University Guri Hospital.

\section{Measures}

\section{Childhood Trauma Questionnaire-Short Form (CTQ-SF)}

The CTQ-SF is a 28-item self-report inventory developed to measure five types of abuse or neglect in childhood or adolescence. ${ }^{15}$ Respondents are queried on items with a 5-point, Likert-type answer format ranging from never true (score=1) to very often true (score=5). Each subscale (i.e., SA, PA, EA, $\mathrm{PN}$ and $\mathrm{EN}$ ) contains five items, and an additional three items are intended to measure any tendency to minimize or deny the abuse (MD subscale). Good to excellent internal consistency for each subscale except PN has been obtained from clinical samples ${ }^{15}$ and community populations. ${ }^{19}$

This scale has also demonstrated good stability over three months among substance abusers ${ }^{14}$ and has shown a significant correlation with therapist rating. ${ }^{15}$ To estimate the prevalence of each kind of childhood maltreatment, we used the cut-off values for moderate or severe maltreatment suggested by Bernstein and Fink. ${ }^{14}$ These cut-off values are based on data from a non-clinical female sample in the U.S. and varies according to CTQ subtype. ${ }^{14}$

\section{Translation of the CTQ}

The original CTQ-SF was translated into Korean by the corresponding author (DK), and two bilingual mental health professionals back-translated the Korean version to assure its translation. These back-translations and the Korean version (CTQ-K) were reviewed and approved by the research and development team at NCS Pearson Inc, the scale copyright owner.

\section{Trauma Antecedent Questionnaire (TAQ)}

Concurrent validity was assessed with the TAQ, originally 
a 42-item self-report measure, which covers 37 traumatic or negative experiences as well as five positive aspects. Respondents were asked to score the severity or intensity of each experience. To parallel and pertain to the CTQ, we used the physical abuse, sexual abuse, emotional abuse, and neglect subscales of the TAQ. The competence and safety subscales were included in the analysis to assess the discriminant validity of the CTQ-K. Further, we limited the developmental period to less than 18 years (age $0-18$ ) to focus on childhood and adolescent experiences.

The Korean version used for this study had not been previously tested for its psychometric properties. In this study, Cronbach's a for 37 items was 0.88 .

\section{Impact of Events Scale-Revised (IES-R)}

To measure convergent validity, the 22-item IES-R questionnaire was also administered. The IES-R assesses symptoms of posttraumatic stress disorder (PTSD) per DSM-IV criteria. PTSD is a representative psychiatric disorder that develops after traumatic events including childhood abuse.

The Korean version (IES-R-K) used in this study demonstrated adequate internal consistency (Cronbach's $\alpha=0.69$ $0.83)$ and high two week test-retest reliability ( $\mathrm{r}=0.88)$ among traumatized individuals. The IES-R-K has also shown adequate concurrent validity with other PTSD measures. ${ }^{20}$

\section{Dissociative Experiences Scale-Taxon (DES-T)}

The DES-T is a brief eight-item version of the original 28item DES to measure pathological dissociation distinct from normal dissociative symptoms such as absorption. This scale is not a diagnostic tool but can be used to screen for pathological dissociative experiences.

The original DES-Korean version demonstrated adequate reliability and validity in a clinical population: high internal consistency (Cronbach's $\alpha=0.94$ ), adequate test-retest reliability $(r=0.64)$, and adequate discriminant validity against non-dissociative psychiatric disorders. The corrected itemto-total correlation coefficients for the eight DES-T items ranged from 0.55 to $0.71 .^{21}$

\section{Statistical analysis}

Chi-square and independent t-tests were used to compare the two samples. We used Pearson's correlation to assess testretest reliability and validity. The internal consistency of the CTQ-K was evaluated using Cronbach's $\alpha$. We also conducted an exploratory principle component analysis with Varimax rotation. The number of factors was determined by the eigenvalues and the variance explained by each factor. All data were analyzed using SPSS 18.0 for Windows.
Table 1. General characteristics of the psychiatric outpatients $(\mathrm{N}=163)$

\begin{tabular}{|c|c|}
\hline Variable & $\mathrm{N}(\%)$ \\
\hline \multicolumn{2}{|l|}{ Sex } \\
\hline Men & $64(39.3)$ \\
\hline Women & $99(60.7)$ \\
\hline \multicolumn{2}{|l|}{ Diagnosis } \\
\hline Anxiety disorder & $69(42.3)$ \\
\hline Mood disorder & $38(23.3)$ \\
\hline Adjustment disorder & $20(12.3)$ \\
\hline Dissociative disorder & $14(8.6)$ \\
\hline Personality disorder & $11(6.7)$ \\
\hline Somatoform disorder & $9(5.5)$ \\
\hline Others & $2(1.2)$ \\
\hline \multicolumn{2}{|l|}{ Marital status } \\
\hline Married & $87(53.4)$ \\
\hline Divorced & $14(8.6)$ \\
\hline Widowed & $5(3.1)$ \\
\hline Never married & $57(35.0)$ \\
\hline \multicolumn{2}{|l|}{ Employment } \\
\hline Employed & $49(30.1)$ \\
\hline Unemployed & $57(35.0)$ \\
\hline Student or wife & $47(28.8)$ \\
\hline \multicolumn{2}{|l|}{ Education } \\
\hline Less than high school & $26(16.0)$ \\
\hline High school graduate & $101(62.0)$ \\
\hline University graduate & $34(20.9)$ \\
\hline Post-graduate school & $1(6.1)$ \\
\hline \multicolumn{2}{|c|}{ Monthly income (10,000 won) } \\
\hline$<200$ & $59(36.2)$ \\
\hline $200-399$ & $51(31.3)$ \\
\hline $400-599$ & $31(19.0)$ \\
\hline$>600$ & $8(4.9)$ \\
\hline \multicolumn{2}{|l|}{ Religion } \\
\hline None & $68(41.7)$ \\
\hline Christian & $39(23.9)$ \\
\hline Buddhism & $30(18.4)$ \\
\hline Catholic & $11(6.7)$ \\
\hline
\end{tabular}

Because of missing data, total number may differ from the original sample size

\section{RESULTS}

\section{Childhood trauma prevalence}

Using the cut-off criteria previously described, ${ }^{14} 29 \%$ (47/163) of the sample reported PA, another 23\% (37/163) SA, 25\% (41/163) EA, 49\% (80/163) EN, and 61\% (99/163) PN (Figure 1). 


\section{Reliability}

The Cronbach's a for the 28-item CTQ-K was excellent $(>0.90)$, and the alpha values for the subscales ranged from $0.68-0.88$ (Table 2). The four-week test-retest reliability of the CTQ-K was also high $(>0.80)$, and the correlation coefficients for the subscales ranged from 0.76 to 0.88 (Table 2).

\section{Concurrent validity}

Total CTQ-K scores were significantly correlated with the emotional abuse, physical abuse, sexual abuse, and neglect subscales of the TAQ. Each subscale of the CTQ was also significantly correlated with the corresponding subscales of the TAQ (Table 3).

\section{Convergent and discriminant validity}

Total CTQ-K scores were significantly correlated with posttraumatic symptoms and pathological dissociation. However, the correlation between the IES-R and the DES-T differed depending on the type of abuse. That is, SA, EN, and PN were not correlated with the IES-R and only the EA was significantly correlated with the DES-T (Table 3).

Total CTQ-K scores were inversely correlated with competence $(\mathrm{r}=-0.28, \mathrm{p}=0.03)$ and safety $(\mathrm{r}=-0.53, \mathrm{p}<0.001)$.

\section{Factorial validity}

Principal component analysis of the sample identified five factors that explained $64.0 \%$ of the total variance: Factor 1 (emotional neglect), Factor 2 (physical abuse), Factor 3 (sex-

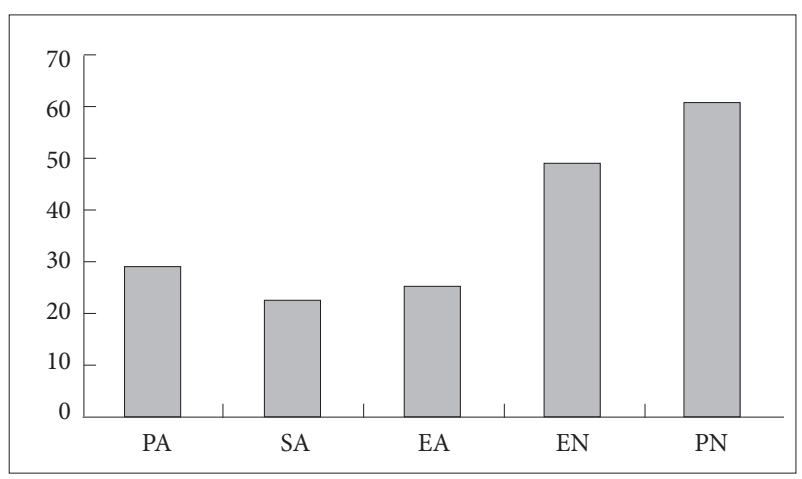

Figure 1. Prevalence of childhood trauma in psychiatric outpatients $(n=163)$. The cut-off criteria used in this figure are from a U.S. non-clinical population and have not been confirmed in Koreans. PA: physical abuse, SA: sexual abuse, EA: emotional abuse, EN: emotional neglect, PN: physical neglect. ual abuse), Factor 4 (emotional abuse), and Factor 5 (physical neglect; Table 4).

\section{DISCUSSION}

In this study, the CTQ-K showed adequate reliability and validity among psychiatric outpatients. First, the internal consistency of the global scale was high. Of note, the Cronbach's $\alpha$ of the subscales varied. For instance, although above the lenient level, the PN scale showed a questionable Cronbach's $\alpha$ of 0.68 . Similar low levels of internal consistency for the PN scale have been observed in other studies. ${ }^{12,14,19}$ This lack of homogeneity in PN may indicate a problem in the original construction. Gerdner and Allgulander ${ }^{12}$ have suggested that poor internal consistency may be due to poor differentiation of PN from EN or because the two separate factors are conceptually intermingled in the construct of physical neglect (i.e., neglect of care and neglect of supervision).

This view was also supported by our factor analysis that showed that two items (no 2: I was taken care of and no 26: I was taken to doctor) in the proposed PN loaded onto the EN subscale. This finding differs from the original factor structure suggested by Bernstein and his colleagues. ${ }^{15}$ However, in the original study, factor loadings for the items constituting PN were relatively lower, and one subsequent study failed to demonstrate factorial validity of the PN subscale. ${ }^{22}$ It is of note that our finding has also been observed in three different studies. ${ }^{12,23,24}$ Further, one study analyzed gender differences and found that this pattern was seen only among men and not among women. ${ }^{24}$ We were unable to replicate gender differences due to a relatively small sample size in our study.

Collectively, the problematic internal consistency of the PN subscale and the item loadings onto PN that differed from the original study ${ }^{15}$ are not indicative of weakness of the CTQ-K but instead of problems in the construct validity of the original version. Additionally, the five factor structure in this study parallels the findings of the original version, ${ }^{14}$ supporting cross-cultural factorial equivalence.

To our knowledge, this study is the first to demonstrate the four-week test-retest reliability of the CTQ-SF. Bernstein and Fink $^{14}$ have reported a similar level of correlation after an interval of 1.6-5.6 months among substance-abusing outpatients. One study using a 53-item version has also reported

Table 2. CTQ-K internal consistency and four-week test-retest reliability

\begin{tabular}{lcccccc}
\hline & EA & PA & SA & EN & PN & CTQ total \\
\hline Cronbach's alpha* & 0.82 & 0.88 & 0.87 & 0.86 & 0.68 & 0.92 \\
Pearson's $\mathrm{r}^{\dagger}$ & 0.76 & 0.88 & 0.72 & 0.83 & 0.78 & 0.87 \\
\hline
\end{tabular}

${ }^{*} \mathrm{~N}=163,{ }^{\dagger} \mathrm{N}=40$. CTQ: Childhood Trauma Questionnaire, EA: emotional abuse, PA: physical abuse, SA: sexual abuse, EN: emotional neglect, PN: physical neglect 


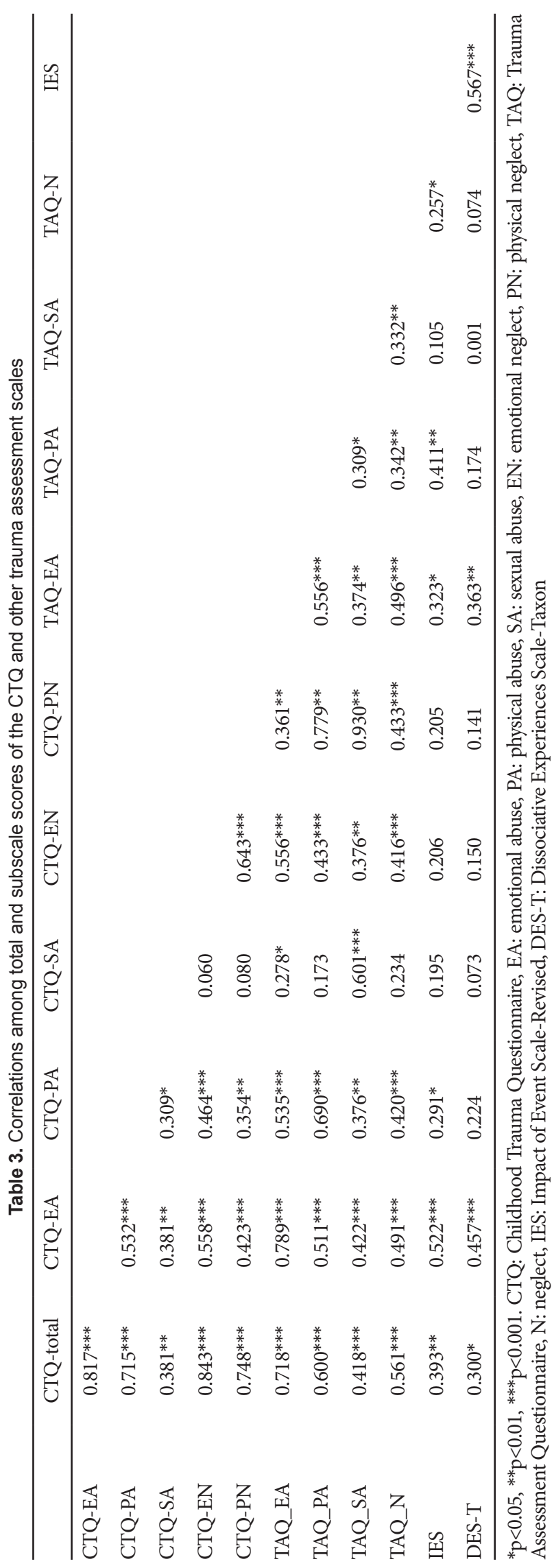

adequate six-month test-retest reliability in outpatients undergoing psychotherapy. ${ }^{25}$ Additionally, another study demonstrated high two-week reliability of the CTQ-SF in college students. ${ }^{23}$ Collectively, these findings indicate that the CTQ is stable over two weeks to six months.

The convergent validity of the CTQ-K was demonstrated by the significant correlation between PTSD symptoms and pathological dissociation, which are representatively and theoretically associated with childhood trauma. ${ }^{26,27}$ The original CTQ-SF also showed a significant correlation with depression, PTSD symptoms, dissociation and alexithymia. ${ }^{13,14}$ In those studies, all five types of childhood trauma were related to depression or PTSD symptoms, while our study did not identify relationships with sexual abuse or neglect. This may be in part due to the small sample size of our study because, although not statistically significant, the modest correlation coefficients obtained were similar to those of previous studies. Another possibility is the low correlation with SA may be because SA may include very mild cases of sexual abuse. ${ }^{28}$ Thus, a dose-dependent response relationship could have influenced the results, although this has not yet been established. Additionally, our finding of a stronger association between emotional abuse and dissociation agrees with a prior study that examined abuse type and dissociative symptoms in patients with conversion. ${ }^{29}$ The CTQ-K demonstrated adequate discriminant validity through negative correlations with competence and safety, which are theoretically judged to be lacking in childhood abuse survivors. ${ }^{30}$

The concurrent validity with another trauma measure indicates that all subtypes of the CTQ-K are substantially associated with the corresponding subscale of the TAQ, with the exception of PN and EN, which show moderate correlations with the neglect scale of the TAQ. This finding likely reflects the conceptual overlap of neglect between both constructs. To our knowledge, this is the first evidence that the CTQ demonstrates convergence with other self-report measures. Previous studies reporting the validity of the CTQ have shown correlations only with therapist rating or interview instruments. ${ }^{15,25}$

Finally, the prevalence of child abuse and neglect in our study was comparable to that reported in previous studies. The highest prevalence was found for PN, followed by EN, PA, EA, and SA. This rank order is largely similar to that reported for the general and clinical populations. ${ }^{31,32}$ However, this current study applied the cut-off scores determined from a non-clinical sample of U.S. women. Thus, cultural and psychometric influences should be considered in interpreting these results. For example, one study has found that the CTQ tends to over-estimate SA and under-estimate PA..$^{28}$ Cultural aspects may play a role in patients' responses to certain items. 
Table 4. Principal component analysis* of the Korean version of the Childhood Trauma Questionnaire ( $N=163)$

\begin{tabular}{|c|c|c|c|c|c|}
\hline \multirow{2}{*}{ Item } & \multicolumn{5}{|c|}{ Factor loading } \\
\hline & 1 & 2 & 3 & 4 & 5 \\
\hline \multicolumn{6}{|l|}{ Factor 1: Emotional neglect } \\
\hline 5. Made to feel important & 0.76 & & & & \\
\hline 28. Family source of strength & 0.73 & 0.33 & & & \\
\hline 19. Family felt close & 0.70 & & & 0.34 & \\
\hline 2. Taken care of & 0.69 & & & & \\
\hline 7. Felt loved & 0.67 & & & & \\
\hline 26. Taken to doctor & 0.66 & & & & 0.32 \\
\hline 13. Looked out for & 0.65 & & & & \\
\hline \multicolumn{6}{|l|}{ Factor 2: Physical abuse } \\
\hline 11. Hit hard enough to leave bruises & & 0.82 & & & \\
\hline 12. Punished with hard objects & & 0.78 & & & \\
\hline 17. Hit hard enough to be noticed & & 0.77 & & & \\
\hline 9. Hit hard enough to see doctor & & 0.76 & & 0.33 & \\
\hline 15. Physically abused & & 0.69 & & & \\
\hline \multicolumn{6}{|l|}{ Factor 3: Sexual abuse } \\
\hline 23. Made to do sexual things & & & 0.88 & & \\
\hline 27. Sexually abused & & & 0.83 & & \\
\hline 20. Touched sexually & & & 0.82 & & \\
\hline 24. Molested & & & 0.74 & & \\
\hline 21. Threatened for something sexual & & & 0.68 & & \\
\hline \multicolumn{6}{|l|}{ Factor 4: Emotional abuse } \\
\hline 14. Family said hurtful things & & & & 0.73 & \\
\hline 8. Unwanted & & & & 0.68 & \\
\hline 3. Called names by family & & 0.38 & & 0.64 & \\
\hline 18. Felt hated by family & & 0.33 & & 0.63 & \\
\hline 25. Emotionally abused & 0.37 & 0.32 & & 0.59 & \\
\hline \multicolumn{6}{|l|}{ Factor 5: Physical neglect } \\
\hline 1. Not enough to eat & & & & & 0.83 \\
\hline 6. Wore dirty clothes & & & & & 0.71 \\
\hline 4. Parents were drunk & & & & & 0.48 \\
\hline Eigenvalue & 3.9 & 3.9 & 3.5 & 3.0 & 1.8 \\
\hline Percent total variance & 15.8 & 15.4 & 13.9 & 11.8 & 7.2 \\
\hline
\end{tabular}

Loadings smaller than 0.30 are not displayed. *varimax rotation with Kaiser normalization

A Turkish study has found that the patterns of response to the PA, EA and EN items are different from those of the English version, indicating cross-cultural differences in interpreting parental behavior and parental emotional expression toward their children. ${ }^{11}$

This study has several limitations. First, the participants were non-psychotic outpatients in a psychiatric clinic of a university hospital, which limits the generalizability of these findings. These findings need to be replicated in a larger study and in other psychiatric populations (e.g, inpatients or those with psychotic illness) and in healthy people in the community. Second, the TAQ was used as comparison tool for concurrent validity and was not tested for its psychometric properties. We were unable to find any validated Korean measures for assessing childhood trauma. The TAQ was chosen for this study because it is one of the few instruments that possess similar types of subscales to the CTQ. Third, the sample size was relatively small for factor analysis, which prevented further data exploration.

Despite these limitations, our study confirmed at least adequate reliability and validity, thus supporting the use of the CTQ-K for Korean-speaking clinical populations. Further work should include different clinical and general population samples and confirmatory factor analysis. Additionally, it is 
important for the validity of cut-off values suggested for the original version to be confirmed for the Korean version.

\section{Acknowledgments}

This work was supported by the research fund of Hanyang University (HY-2010-I).

\section{REFERENCES}

1. Goodwin J, Attias R, McCarty T, Chandler S, Romanik R. Reporting by adult psychiatric patients of childhood sexual abuse. Am J Psychiatry 1988;145:1183-1184.

2. Mitchell D, Grindel CG, Laurenzano C. Sexual abuse assessment on admission by nursing staff in general hospital psychiatric settings. Psychiatr Serv 1996;47:159-164.

3. Jacobson A, Koehler JE, Jones-Brown C. The failure of routine assessment to detect histories of assault experienced by psychiatric patients. Hosp Community Psychiatry 1987;38:386-389.

4. Mueser KT, Salyers MP, Rosenberg SD, Goodman LA, Essock SM, Osher FC, et al. Interpersonal trauma and posttraumatic stress disorder in patients with severe mental illness: demographic, clinical, and health correlates. Schizophr Bull 2004;30:45-57.

5. Jacobson A. Physical and sexual assault histories among psychiatric outpatients. Am J Psychiatry 1989;146:755-758.

6. Swett C Jr, Surrey J, Cohen C. Sexual and physical abuse histories and psychiatric symptoms among male psychiatric outpatients. Am J Psychiatry 1990;147:632-636.

7. Brown GR, Anderson B. Psychiatric morbidity in adult inpatients with childhood histories of sexual and physical abuse. Am J Psychiatry 1991;148:55-61.

8. Read J, Hammersley P, Rudegeair T. Why, when and how to ask about childhood abuse. Adv Psychiatr Treat 2007;13:101-110.

9. Bernstein DP, Fink L, Handelsman L, Foote J, Lovejoy M, Wenzel K, et al. Initial reliability and validity of a new retrospective measure of child abuse and neglect. Am J Psychiatry 1994;151:1132-1136.

10. Burgermeister D. Childhood adversity: a review of measurement instruments. J Nurs Meas 2007;15:163-176.

11. Aslan H, Alparslan ZN. Initial validity and reliability of the Turkish version of the Childhood Trauma Questionnaire. Ann Med Sci 2000; 9:113-119.

12. Gerdner A, Allgulander C. Psychometric properties of the Swedish version of the Childhood Trauma Questionnaire-Short Form (CTQSF). Nord J Psychiatry 2009;63:160-170.

13. Wingenfeld K, Spitzer C, Mensebach C, Grabe HJ, Hill A, Gast U, et al. The German version of the Childhood Trauma Questionnaire (CTQ): preliminary psychometric properties. Psychother Psychosom Med Psychol 2010;60:442-450.

14. Bernstein DP, Fink L. Childhood Trauma Questionnaire. A Retrospective Self-Report. Manual. San Antonio, TX: The Psychological Corporation, Harcourt Brace \& Company; 1998.

15. Bernstein DP, Stein JA, Newcomb MD, Walker E, Pogge D, Ahluvalia T, et al. Development and validation of a brief screening version of the
Childhood Trauma Questionnaire. Child Abuse Negl 2003;27:169-190.

16. Herman JL, Perry JC, van der Kolk BA. Childhood trauma in borderline personality disorder. Am J Psychiatry 1989;146:490-495.

17. Weiss DS, Marmar CR. The Impact of Event Scale-Revised. In: Wilson JP, Keane TM, Editors. Assessing Psychological Trauma and PTSD. New York: Guilford Press. 1997, p.399-411.

18. Waller NG, Putnam FW, Carlson EB. Types of dissociation and dissociative types: a taxometric analysis of dissociative experiences. Psychol Methods 1996;1:300-321.

19. Scher CD, Stein MB, Asmundson GJ, McCreary DR, Forde DR. The childhood trauma questionnaire in a community sample: psychometric properties and normative data. J Trauma Stress 2001;14:843-857.

20. Eun HJ, Kwon TW, Lee SM, Kim TH, Choi MR, Cho SJ. A study on reliability and validity of the Korean version of impact of event scalerevised. J Korean Neuropsychiatr Assoc 2005;44:303-310.

21. Park JM, Choe BM, Kim M, Han HM, Yoo SY, Kim SH, et al. Standardization of dissociative experiences scale-Korean version. Korean J Psychopathol 1995;4:105-125.

22. Villano CL, Cleland C, Rosenblum A, Fong C, Nuttbrock L, Marthol $\mathrm{M}$, et al. Psychometric utility of the Childhood Trauma Questionnaire with female street-based sex workers. J Trauma Dissociation 2004;5: 33-41.

23. Garrusi B, Nakhaee N. Validity and reliability of a Persian version of the Childhood Trauma Questionnaire. Psychol Rep 2009;104:509-516.

24. Wright KD, Asmundson GJ, McCreary DR, Scher C, Hami S, Stein MB. Factorial validity of the Childhood Trauma Questionnaire in men and women. Depress Anxiety 2001;13:179-183.

25. Paivio SC. Stability of retrospective self-reports of child abuse and neglect before and after therapy for child abuse issues. Child Abuse Negl 2001;25:1053-1068.

26. Sullivan TP, Fehon DC, Andres-Hyman RC, Lipschitz DS, Grilo CM. Differential relationships of childhood abuse and neglect subtypes to PTSD symptom clusters among adolescent inpatients. J Trauma Stress 2006;19:229-239.

27. Chu JA, Dill DL. Dissociative symptoms in relation to childhood physical and sexual abuse. Am J Psychiatry1990;147:887-892.

28. DiLillo D, Fortier MA, Hayes SA, Trask E, Perry AR, Messman-Moore $\mathrm{T}$, et al. Retrospective assessment of childhood sexual and physical abuse: a comparison of scaled and behaviorally specific approaches. Assessment 2006;13:297-312.

29. Sar V, Islam S, Oztürk E. Childhood emotional abuse and dissociation in patients with conversion symptoms. Psychiatry Clin Neurosci 2009; 63:670-677.

30. Levin PA, Kline M. Trauma through a Child's Eyes: Awakening the Ordinary Miracle of Healing. California: North Atlantic Books; 2007.

31. Akyuz G, Sar V, Kugu N, Dogan O. Reported childhood trauma, attempted suicide and self-mutilative behavior among women in the general population. Eur Psychiatry 2005;20:268-273.

32. Spertus IL, Yehuda R, Wong CM, Halligan S, Seremetis SV. Childhood emotional abuse and neglect as predictors of psychological and physical symptoms in women presenting to a primary care practice. Child Abuse Negl 2003;27:1247-1258. 